\title{
Conceptual Framework for an Integrated Method to Optimize Sustainability of Engineering Systems
}

\author{
Alfredo del Caño, María Pilar de la Cruz, Juan José Cartelle and Manuel Lara \\ Department of Industrial Engineering II, Higher Polytechnic University College, University of Coruña, Campus of Esteiro, Ferrol \\ 15403, Spain
}

Received: April 11, 2015 / Accepted: June 03, 2015 / Published: July 31, 2015.

\begin{abstract}
It is necessary to change the current dynamic of growth, to assure that future generations can satisfy their needs. Sustainable development and global sustainability are two concepts that have achieved great importance in almost every sector of activity. There is a wide range of methods and models for sustainability assessment. Nevertheless, it is necessary to go beyond evaluation, looking for sustainability optimization. In spite of this, little work has been done on the latter field. The authors present here a conceptual proposal for an integrated method to optimize sustainability of engineering systems, based on the MIVES (integrated value method for evaluating sustainability) method. For a better understanding of the method, its essential steps for optimizing an energy sub-system are summarized (shell-and-tube heat exchanger).
\end{abstract}

Key words: Sustainability, MIVES, simulation, optimization, metaheuristics.

\section{Introduction}

It is currently accepted that there are limits to growth. For this reason, measures are starting to be taken in order to protect the current and future generations from the consequences of overcoming those limits.

Certain terms and concepts have arisen from this trend. Over the years, they have acquired a high degree of notoriety in almost any sphere of activity. In particular, the terms sustainable development and sustainability come to mind. On the whole, these terms can be related to the capacity to do something with minimum or nil impact on the planet and its population. Unfortunately, almost every activity has some kind of effect on its surroundings. For the moment, it is therefore impossible to achieve full-scale, strict sustainable development. Perhaps it could be reached in the future, with the help of new technologies.

As stated in the Rio Declaration on the Environment

Corresponding author: Juan José Cartelle, Ph.D. student, research fields: sustainability assessment of power plants, sustainability optimization of energy systems and energy economics. E-mail: juan.cartelle1@udc.es. and Development [1], human beings are said to have the right to a healthy and productive life in harmony with nature. This involves aspects related to the economy (productive life), society (healthy life) and the environment (in harmony with nature). Therefore, it may be claimed that, sustainable development lies on three basic pillars: environmental, social and economic. Some authors consider a fourth pillar, the technical or technical-functional one.

Aspects relating to the environment include pollution control and a reduced consumption of energy, materials, or other natural resources. Economic factors are related to equality and development in this field, in turn, linked to the productivity of the planet's resources in the long term. Social concerns are related to population's health and comfort, ensuring a decent life and social stability for people. Technical aspects are related to functional or technical-technological advantages and disadvantages of the engineering systems under development. Table 1 lists potential indicators that can be included in a sustainability assessment in the energy sector. They are a selection of 
all the sustainability aspects found in Refs. [2, 3], among many others. There are different methods for sustainability assessment. Environmental LCA (life-cycle analysis) is probably the most popular one, the various impacts on the planet are estimated, taking into account all the phases of a product's life-cycle from cradle to grave, or even from cradle to cradle. LCA uses measurable variables called indicators. The use of LCA has been extended to the economic and social fields. Many authors have performed LCA studies in the energy sector. Among many others, Kannan, et al. [4] made a life cycle assessment (including a life cycle cost analysis) to quantify the non-renewable energy use and global warming potential in electricity generation from an oil fired steam turbine plant in Singapore. Odeh and Cockerill [5] examined the life cycle greenhouse gas emissions from existing pulverized coal power plants in the UK (United Kingdom). In Ref. [6], the author presents the results of a LCA of photovoltaic energy generation. Varun, et al. [7] made a review of existing life cycle analyses of renewable sources based electricity generation systems. The results of LCA can be used directly, but there are methods for integrating the different indicators' assessments.

The majority of integration methods currently used in the construction sector are based on a weighted scoring system for different sustainability indicators. Research is being done at the moment on more sophisticated alternatives, such as the AHP (analytic hierarchy process), the MIVES (integrated value method for evaluating sustainability) method [8], or fuzzy mathematics.

As pointed out above, the sustainability literature is mainly focused on the assessment, particularly in environmental LCA. It is now necessary to go further, looking for sustainability optimization, for maximizing the contribution to sustainable development of engineering systems. Despite this, as far as the authors know, optimization has received almost no attention so far.

There are not published studies about the optimization of sustainability assessment models based on the MIVES method. In fact, there are very few published studies that discuss the sustainability optimization from a general point of view, and none of them cover with enough depth the four sustainability pillars previously mentioned.

The authors are working in the optimization of MIVES assessment models, applied to the energy and construction sectors. The aim of this paper is to present the main ideas and techniques to establish such methodology, using an energy sub-system (shell-and-tube heat exchanger) as an example of product to be optimized.

\section{The MIVES Method and Its Application to Sustainability Assessment}

The MIVES method is a combination of techniques based on a requirement tree [8,9], value functions [10], and the AHP $[11,12]$. MIVES is used to transform different types of variables, measured with different

Table 1 Sustainability indicators in the energy sector.

\begin{tabular}{ll}
\hline Dimension & Indicators \\
\hline \multirow{2}{*}{ Environmental } & $\begin{array}{l}\text { Global warming, depletion of the ozone layer, acidification, ionizing radiation, eutrophication, heavy metals, } \\
\text { carcinogens, winter smog, summer smog, conventional waste generation, special waste generation, high-level } \\
\text { waste generation, intermediate-level and low-level waste generation, sterile waste generation, available reserves of } \\
\text { fuel and raw materials, land use, noise, bad odours, impact geographical range. }\end{array}$ \\
\hline Social & $\begin{array}{l}\text { Employment generation, population displacement, social benefits, risk of construction accidents, risk of operation } \\
\text { and maintenance accidents, risk of external accidents, visual impact, social acceptability, effect on public budget. }\end{array}$ \\
\hline Economic & $\begin{array}{l}\text { Mining and extraction cost, pre-treatment and enrichment cost, transportation cost, engineering cost, process } \\
\text { equipment cost, cost of civil works, cost of fuel and } \mathrm{CO}_{2} \text { emissions rights, operation and maintenance cost, } \\
\text { decommissioning cost, subsidies. }\end{array}$ \\
\hline $\begin{array}{l}\text { Technical- } \\
\text { functional }\end{array}$ & $\begin{array}{l}\text { Reliability of electricity supply, variability/regularity of electricity supply, stability of the power supply chain, } \\
\text { uncertainty in generation, generation manageability, maturity. }\end{array}$ \\
\hline
\end{tabular}


units, in the same dimensionless unit. It makes it possible to consider non-linearity in the assessment. Moreover, it takes into account the relative importance of the different aspects considered in the evaluation. Finally, it helps to integrate environmental, social, economic and technical indicators in a single, global sustainability index. MIVES is composed of seven phases, which will be described below.

In Phase A, the problem to be solved will be defined. This problem usually entails designing an engineering system in line with sustainability criteria. In fact, MIVES can be used for help in other type of systems or decisions.

In Phase B, the requirement tree will be constructed. This tree is a hierarchical scheme in which the different characteristics of the product or process to be assessed are defined in an organized way. It normally has three levels: requirements, criteria and indicators. Fig. 1 includes an example of this kind of graph.

The third level defines the concrete characteristics that are going to be assessed (indicators). The other two levels establish a structure to break down the requirements. The purpose of the tree is two-fold. On the one hand, the problem is structured to organize the aspects collected in the model and provide a general view of the problem. On the other hand, the tree later facilitates establishing the weightings of the indicators, and calculating the global sustainability index.

In Phase $\mathrm{C}$, the value functions will be established. Here, mathematical elements from the general decision-making theory come into play. In particular, general aspects from MCDM (multi-criteria decision making) are used.

When a design alternative is compared to others, it is possible to consider the existence of a value function $V$ : $P \rightarrow R$, with $P=\left(P_{1}, P_{2}, \ldots, P_{N}\right)$ the set of indicators included in the tree. The problem consists of constructing a dimensionless value function $V(P)$, which integrating all the criteria $P_{i}$, reflects the preferences of the decision makers. The solution is a function $V$, which is the weighted sum of the $N$ functions for value $V_{i}$ corresponding with the $N$ indicators. For a requirement tree with three levels, the $V$ function takes the shape of Eq. (1):

$$
V(P)=\sum_{i=1}^{i=N} \alpha_{i} \cdot \beta_{i} \cdot \gamma_{i} \cdot V_{i}\left(P_{i}\right)
$$

$V(P)$ measures the degree of sustainability of the alternative under assessment, with respect to the set of indicators $P ; \alpha_{i}$ and $\beta_{i}$ are the weights of the requirements and criteria to which each indicator $i$ belongs to; $\gamma_{i}$ are the weights for the different indicators; $V_{i}\left(P_{i}\right)$ are the value functions used to measure the degree of sustainability of the alternative under study with respect to a given indicator $i$; and $N$ is the total number of indicators taken into account in the assessment. MIVES uses Eq. (2) as a basis for defining each value function $V_{i}$.

$$
V_{i}=\frac{1-e^{-m_{i}\left(\frac{\left|P_{i}-P_{i, \min }\right|}{n_{i}}\right)^{A_{i}}}}{1-e^{-m_{i}\left(\frac{\left|P_{i, \max }-P_{i, \min }\right|}{n_{i}}\right)^{A_{i}}}}
$$

In Eq. (2), $P_{i}$ is the input value of the indicator $i$ for the alternative under study, $P_{i, \max }$ and $P_{i, \min }$ are, respectively, the values of $P_{i}$ associated to the maximum and minimum levels of sustainability (here 1 and 0$) . A_{i}, n_{i}$ and $m_{i}$ are shape factors used to generate concave, convex, S-shaped or straight line value functions. The different geometries make it possible to establish greater or lesser exigency when complying with the requisites for satisfying a given indicator. The denominator from Eq. (2) ensures that $V_{i}$ returns values that fall within the interval $[0,1]$. Fig. 2 includes an example of value function for an indicator related to ecopoints of environmental impact that measures the different kinds of environmental impact, such as global warming, depletion of the ozone layer, acidification, among others.

Step functions can be used for qualitative variables. These are discrete, stepped functions in which each tier is associated with a possible situation or semantic label. 


\begin{tabular}{|c|c|c|c|c|c|c|}
\hline \multirow{18}{*}{ 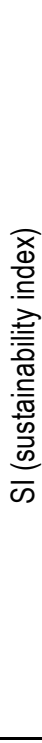 } & \multicolumn{2}{|c|}{ Requirements } & \multicolumn{2}{|r|}{ Criteria } & \multicolumn{2}{|r|}{ Indicators } \\
\hline & \multirow{8}{*}{$28.01 \%$} & \multirow{8}{*}{ Economic } & $21.27 \%$ & Cost of obtaining the fuel or raw materials & $100.00 \%$ & Mining and extraction cost \\
\hline & & & $8.59 \%$ & Cost of preparing the fuel or raw materials & $100.00 \%$ & Pre-treatment and enrichment cost \\
\hline & & & $11.22 \%$ & Cost of transporting the fuel or raw materials & $100.00 \%$ & Transportation cost \\
\hline & & & \multirow{2}{*}{$32.32 \%$} & \multirow{2}{*}{ Investment cost } & $25.00 \%$ & Engineering and civil works cost \\
\hline & & & & & $75.00 \%$ & Process equipment cost \\
\hline & & & \multirow{2}{*}{$24.19 \%$} & \multirow{2}{*}{ Operating cost } & $42.23 \%$ & Cost of fuel and $\mathrm{CO}_{2}$ \\
\hline & & & & & $57.77 \%$ & Operation and maintenance cost \\
\hline & & & $2.41 \%$ & Subsidies & $100.00 \%$ & Subsidies \\
\hline & \multirow{5}{*}{$39.30 \%$} & \multirow{5}{*}{ Social } & $20.11 \%$ & Employment generation & $100.00 \%$ & Employment generated \\
\hline & & & $8.02 \%$ & Population displacement caused by the project & $100.00 \%$ & Population displacement \\
\hline & & & $8.02 \%$ & Development of new areas & $100.00 \%$ & Development of new areas \\
\hline & & & $61.17 \%$ & Health and safety & $100.00 \%$ & Risk of accident \\
\hline & & & $2.69 \%$ & Visual impact & $100.00 \%$ & Visual impact \\
\hline & \multirow{4}{*}{$32.69 \%$} & \multirow{4}{*}{ Environmental } & $88.37 \%$ & Environmental impact & $100.00 \%$ & Ecopoints of environmental impact \\
\hline & & & \multirow{2}{*}{$4.86 \%$} & \multirow{2}{*}{ Discomfort associated with noise and odours } & $60.00 \%$ & Noise \\
\hline & & & & & $40.00 \%$ & Bad odours \\
\hline & & & $6.77 \%$ & Impact geographical range & $100.00 \%$ & Local/regional/global impact \\
\hline
\end{tabular}

Fig. 1 Example of a requirement tree.

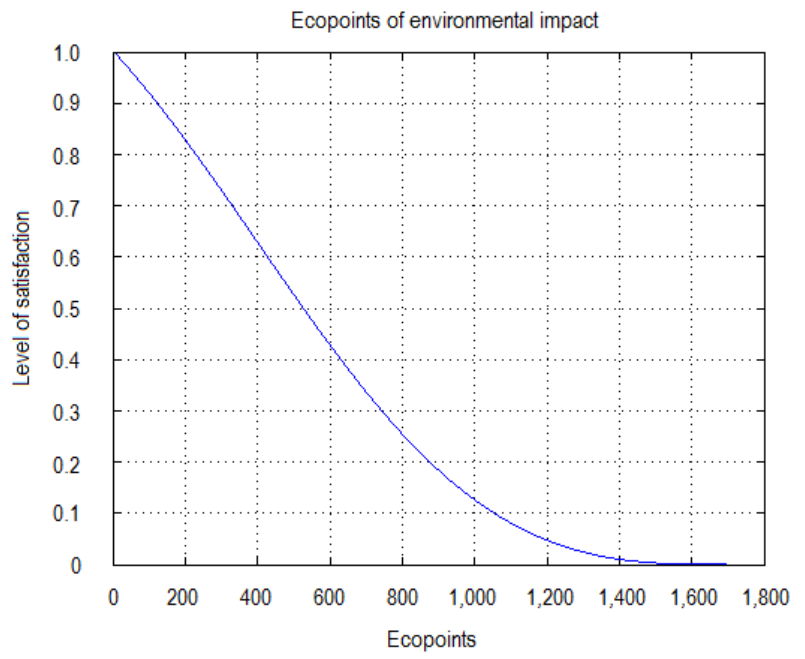

Fig. 2 Example of value function for an indicator related to ecopoints of environmental impact.

In Phase $\mathrm{D}$, the weights $\alpha_{i}, \beta_{i}$ and $\gamma_{i}$ will be established for the various branches on the requirement tree. In some cases, this can be done directly. In general, directly allocating weights in branches with up to four indicators does not generate problems. With more than four, one often loses the overall view and this can lead to inconsistencies, among other potential problems. It is a good idea to use AHP [9, 11, 12]. This technique helps to organize the process efficiently, reduce its complexity and subjectivity and decrease possible disagreements between the team members. AHP is based on a pairwise comparison of the relative importance for the various branches radiating from the same point of the tree. Phases $\mathrm{C}$ and $\mathrm{D}$ can be developed simultaneously.

Phase E consists of defining different design alternatives that will be evaluated by means of the model. Those options will be assessed in Phase F, calculating the sustainability index for each one. Eq. (1) will be applied for this purpose. Finally, in Phase G, decisions will be made, and the designers will choose the best option. Additional information about MIVES can be found in Refs. [8, 9].

\section{Optimizing the Sustainability of Engineering Systems}

\subsection{Dealing with Uncertainty in MIVES Models}

Uncertainty can affect specific variables of engineering systems, and so the sustainability indicators. Moreover, it could be discrepancies among the experts at the time of establishing value functions and the weights of the tree.

MIVES is a deterministic method, so it does not allow one to consider the uncertainty that could affect the variables included in sustainability assessment models. It is necessary to combine MIVES with a 
technique capable of considering the uncertainty. An option is Monte Carlo simulation [13].

The MIVES-Monte Carlo method is composed of nine phases. In Phase P1, the probabilistic parameters of the MIVES model will be identified. Indicators, weights and value function parameters could be treated as probabilistic variables. It is recommended that, only those variables with the greatest influence over the model and a high degree of uncertainty are established as probabilistic. To this end, a sensitivity analysis can be performed on the deterministic MIVES model.

In Phases P2 and P3, the deterministic (Phase P2) and probabilistic (Phase P3) parameters of the model will be estimated. Phase P2 usually does not cause problems because deterministic variables can be estimated using expert judgement, historical databases would be very helpful.

On the contrary, there are no databases covering the complete set of sustainability indicators used in this kind of models, nor are there any databases related to weights and value functions. Consequently, simple and easy to understand probability distributions should be used: open and closed triangular ones, uniform distributions, Bernoulli and general discrete functions [9], for the potential use of each type of distribution.

In Phases P4-P6, simulation will be performed. According to the previously defined distribution functions, pseudo-random values will be generated for every probabilistic variable (Phase P4). PRNGs (pseudo-random number generators) and complementary techniques will be used for this purpose, for instance, the inversion (inverse transform method) and acceptance-rejection techniques can be employed [9].

There could be correlations between the model indicators. If possible, it is recommended to use analytical formulae for modelling correlations. When the correlations are not known in an analytical way, the technique called "correlation among samples" could be used [9].

Eqs. (1) and (2) will be applied in each iteration, to obtain a potential value for the final sustainability index of the alternative under study (Phase P5). Phases P4 and P5 will be repeated until convergence has been reached in the results (Phase P6).

When a value function is being constructed, discrepancies about of its geometry may appear among the experts. One option, in Phase P3, is to define two or more value functions for that indicator. A specific probability will then be assigned to each one of those functions. Another alternative could be estimated distribution functions for $A_{i}, m_{i}$ and $n_{i}$, in Phase P3.

Uncertainty or disagreements may exist about the weights $\gamma_{i}, \beta_{i}$ and $\alpha_{i}$. In the first instance, discrepancies could be solved using conventional AHP. In other instance, or in case of uncertainty, distribution functions can be established for the weights.

In Phase P7, a statistical analysis of the output sample will be performed. This means calculating its essential statistical parameters (maximum, minimum, mode, typical deviation, percentiles, etc.), as well as the frequency histogram and the curve of cumulative probability for the global sustainability index.

In Phase P8, the users must interpret the statistical analysis, and make the opportune decisions about the system's design.

Finally, Phase P9 is crucial for improving effectiveness in subsequent applications of the method. Real, final data must be collected, to be used in future projects. Historical databases will make it possible to perfect the model and to estimate its variables more effectively, in the future. Additional information about the MIVES-Monte Carlo method can be found in Ref. [9].

\subsection{The Models to be Optimized, Potential Applications}

The potential models for optimizing complete energy systems (e.g., an air conditioning system, a power plant) are excessively complex for a first work. For the moment, the applications should begin with simpler models related to relatively uncomplicated energy sub-systems, with a limited amount of design parameters. 
As an example, in this section, some ideas about the optimization of a MIVES model applied to a STHE (shell-and-tube heat exchanger) are proposed. Nevertheless, the same concepts can be applied to other partial or complete energy systems.

The principal components of a STHE are shell, shell cover, tubes, channel, channel cover, tubesheet, baffles and nozzles. Other components include tie-rods and spacers, pass partition plates, impingement plate, longitudinal baffle, sealing strips, supports and foundation.

When a STHE is being designed, certain requirements have to be met: flow rates, inlet and outlet temperatures, operating pressure, allowable pressure drop, or heat duty, among others. Depending on the STHE application, the designer may have more or less freedom during the design process. However, normally there are several suitable design alternatives for a particular application. Tube size, material of construction, shell diameter, number of tubes, heat transfer area, tube pitch, tube layout angle, tube layout patterns, baffle type and baffle spacing are design parameters chosen by the designer.

It is possible to create a mathematical model including all the parameters and formulas necessary for an adequate design of a STHE (STHE sub-model). A distribution function can be assigned to the opportune parameters. Specific formulae must be incorporated to the model in order to avoid absurd designs. In this way, a right STHE for the application is ensured, and a valid STHE will be generated in each iteration.

A MIVES model can be also created for evaluating the sustainability index of the STHE (sustainability sub-model). This model will be made up of a set of environmental, social, economic and technical indicators (an appropriate selection of those presented in Table 1, among other suitable indicators).

The STHE and sustainability sub-models must then be linked, configuring the complete model to be optimized. Fig. 3 shows a conceptual graph of this model. Each STHE design will have its own life-cycle energy and resources consumption, its own life-cycle $\mathrm{CO}_{2}, \mathrm{SO}_{2}$ and $\mathrm{PO}_{4}{ }^{-3}$ emissions, among others. The opportune formulae will be established for estimating the sustainability indicators corresponding to each result of the STHE sub-model. These data will be the input values to the MIVES sub-model.

Finally, a conventional or metaheuristics simulation process will be performed, to find the combination of the STHE design parameters with the highest sustainability index.

\subsection{Optimization Techniques}

Frequently MIVES models include discrete variables, so it is not possible to apply the usual derivation techniques to obtain the maximum of the set of mathematical functions defining that kind of models. Other kinds of techniques must be used [14].

The easiest conceptual alternative is to apply Monte Carlo or Latin Hypercube (stratified Monte Carlo) techniques to the model, which simply carry out a random search in a similar way than the one explained here in Section 3.1. After simulation, the alternative with the highest sustainability index will be identified. Since Monte Carlo is an approximate method, this will allow to obtain an exact (optimal) or, more frequently, approximate (suboptimal) solution to the problem.

Metaheuristics [14, 15] are also random exploration algorithms, but they perform a smarter search for that optimal or suboptimal solution, consequently shortening the computational time. Instead of generating random numbers and waiting to the end to identify the optimal, metaheuristics draw conclusions from the intermediate results obtained during the simulation, in order to guide the search towards more promising areas of the solution space. There is a wide range of metaheuristic algorithms [14].

Each type of algorithm has different characteristics, and may not be suitable for some of the possible models to be optimized. Each metaheuristic technique must also be adapted to the model, defining the various operators, parameters and criteria for effectively 


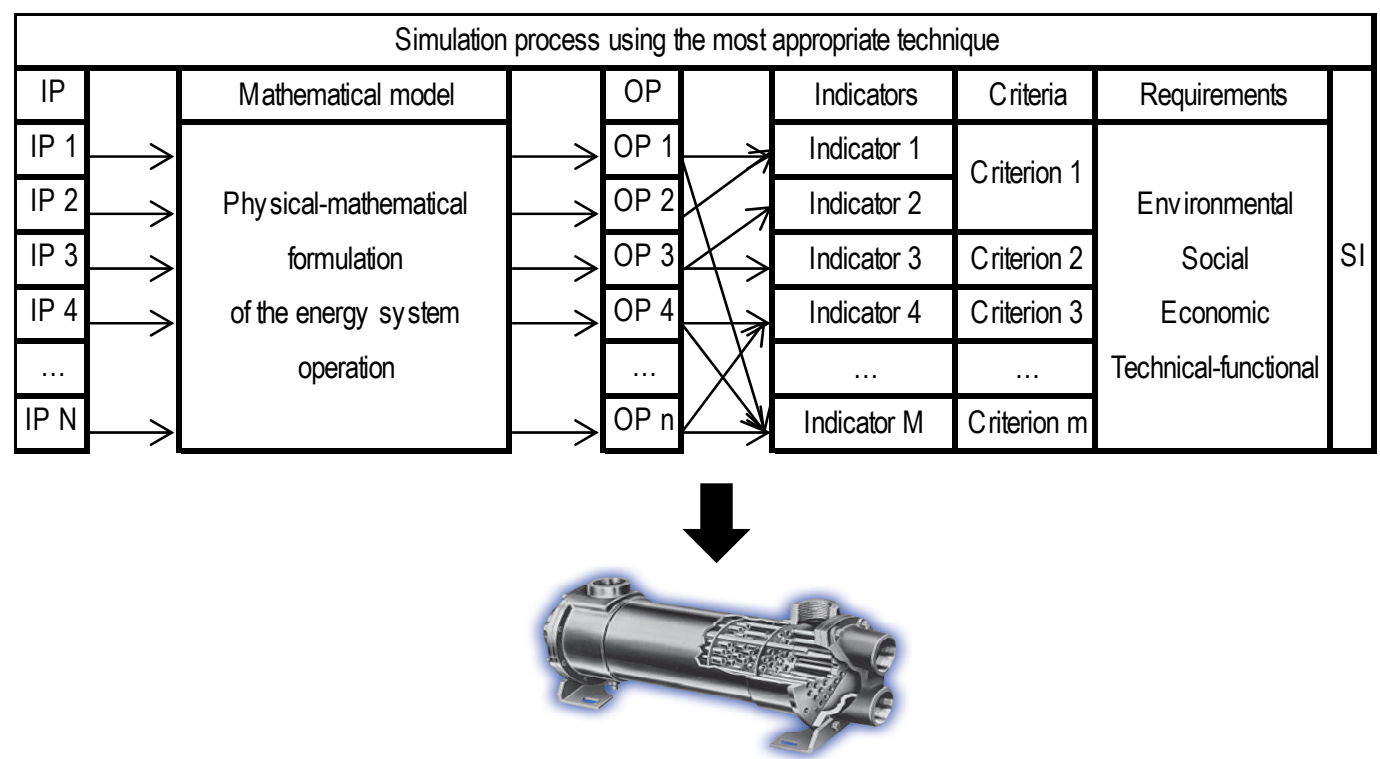

Fig. 3 Conceptual graph representing the models to be optimized.

guiding the search. Genetic algorithms [16, 17], tabu search [18, 19] and simulated annealing [20, 21] may be suitable techniques for this purpose.

\section{Conclusions}

This paper has proposed the main ideas and techniques to establish a methodology for optimizing the sustainability of engineering systems, particularly of energy systems. Since the opportune MIVES models use to include discrete variables, it is not possible to apply conventional derivation techniques. Simulation or metaheuristic techniques must be used. For large engineering systems metaheuristic algorithms will be necessary, in order to shorten the computational time.

As far as the authors know, there are no studies on the most appropriate optimization techniques for the intended purpose. Consequently, it will be necessary to carry out an exploratory phase to select and configure the most suitable metaheuristic technique. At present, it seems that, tabu search, simulated annealing and, above all, genetic algorithms may be suitable techniques for the purpose explained here.

For the moment, the applications should begin with relatively uncomplicated energy sub-systems, with a limited amount of design parameters. Thus, corrections and validation will be easier to be made, increasing the probability of success in larger applications.

\section{References}

[1] United Nations Organization. 1992. "Rio Declaration on Environment and Development." Presented at the UNCED (United Nations Conference on Environment and Development), Río of Janeiro, Brazil. Accessed January 16 ,

2014.

http://www.un.org/documents/ga/conf151/aconf15126-1a nnex1.htm.

[2] Afgan, N. H., and Carvalho, M. G. 2002. "Multi-criteria Assessment of New and Renewable Energy Power Plants.” Energy 27 (August): 739-55.

[3] Kaya, T., and Kahraman, C. 2010. "Multicriteria Renewable Energy Planning Using an Integrated Fuzzy VIKOR \& AHP Methodology: The Case of Istanbul." Energy 35 (June): 2517-27.

[4] Kannan, R., Tso, C. P., Osman, R., and Ho, H. K. 2004. "LCA-LCCA of Oil Fired Steam Turbine Power Plant in Singapore." Energy Conversion and Management 45 (November): 3093-107.

[5] Odeh, N. A., and Cockerill, T. T. 2008. "Life Cycle Analysis of UK Coal Fired Power Plants." Energy Conversion and Management 49 (February): 212-20.

[6] Stoppato, A. 2008. "Life Cycle Assessment of Photovoltaic Electricity Generation." Energy 33 (February): 224-32.

[7] Varun, B. I. K., and Prakash, R. 2009. "LCA of Renewable Energy for Electricity Generation Systems-A Review." Renewable and Sustainable Energy Reviews 3 (June): 1067-73.

[8] Gómez, D., del Caño, A., de la Cruz, M. P., and Josa, A. 
2012. "Generic Methodology for the Assessment of Sustainability of Construction Systems. The MIVES Method." In Sustainability and Construction, edited by Aguado, A. Madrid: Spanish Structural Concrete Association.

[9] de la Cruz, M. P., Castro, A., del Caño, A., Gómez, D., Lara, M., and Cartelle, J. J. 2015. "Comprehensive Methods for Dealing with Uncertainty in Assessing Sustainability. Part I: the MIVES-Monte Carlo Method." In Soft Computing Applications for Renewable Energy and Energy Efficiency, edited by García-Cascales, M. S., Sánchez-Lozano, J. M., Masegosa, A. D., and Cruz-Corona, C. Hershey: IGI-Global.

[10] Alarcón, B., Aguado, A., Manga, R., and Josa, A. 2011. "A Value Function for Assessing Sustainability: Application to Industrial Buildings." Sustainability 3 (January): 35-50.

[11] Saaty, T. 1980. The Analytic Hierarchy Process. New York: McGraw-Hill.

[12] Saaty, T. 2006. Fundamentals of Decision Making and Priority Theory with the Analytic Hierarchy Process.
Pittsburg: RWS Publications.

[13] Ripley, B. D. 1987. Stochastic Simulation. New York: Wiley \& Sons.

[14] Floudas, C. A., and Pardalos, P. M. 2009. Encyclopedia of Optimization. USA: Springer.

[15] Rothlauf, F. 2011. Design of Modern Heuristics: Principles and Application. Mainz: Springer.

[16] Haupt, R. L., and Haupt, S. E. 2004. Practical Genetic Algorithms. Hoboken: Wiley.

[17] Aboshosha, A., and Khalyfa, Y. 2012. Genetic Algorithms Theories and Applications. Saarbrücken: LAP Lambert.

[18] Glover, F. 1989. “Tabu Search: Part I.” ORSA J Comput 1 (February): 190-260.

[19] Glover, F. 1989. "Tabu Search: Part II." ORSA J Comput 2 (November): 4-32.

[20] Kirkpatrick, S., Gelatt, C. D., and Vecchi, M. P. 1983. "Optimization by Simulated Annealing." Science 220 (May): 671-80.

[21] Dekkers, A., and Aarts, E. 1991. "Global Optimization and Simulated Annealing." Mathematical Programming 50 (March): 367-93. 